\title{
PLOD3 suppression exerts an anti-tumor effect on human lung cancer cells by modulating the PKC-delta signaling pathway
}

\author{
Jeong-Hwa Baek ${ }^{1,2}$, Hong Shik Yun ${ }^{1}$, Gyoo Taik Kwon', Janet Lee ${ }^{1}$, Ju-Young Kim¹, Yunhui Jo ${ }^{1}$, Jae-Min Cho',

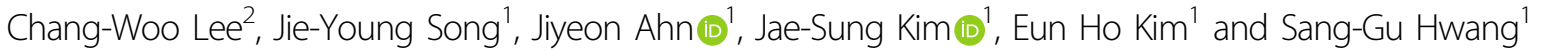

\begin{abstract}
Current lung cancer treatments are far from satisfactory; thus, finding novel treatment targets is crucial. We recently identified procollagen-lysine, 2-oxoglutarate 5-dioxygenase 3 (PLOD3), which is involved in fibrosis and tissue remodeling as a radioresistance-related protein in lung cancer cells; however, its mechanism is unclear. In this study, we designed human PLOD3-specific short interfering (si)RNAs and tested their effects on tumor growth inhibition in vitro and in vivo. PLOD3 knockdown overcame chemoresistance and decreased radioresistance by inducing caspase-3-dependent apoptosis in lung cancer cells. Furthermore, PLOD3 interacted with PKC $\delta$ to activate caspase-2, 4-dependent apoptosis through ER-stress-induced IRE1a activation and the downstream unfolded-protein response pathway. In a mouse xenograft model, PLOD3 knockdown promoted radiation-induced tumor growth inhibition, without side effects. Moreover, lung cancer patients with high PLOD3 expression showed poorer prognosis than those with low PLOD3 expression upon radiotherapy, suggesting that PLOD3 promotes tumor growth. Therefore, PLOD3 siRNA suppresses radioresistance and chemoresistance by inducing apoptosis and renders PLOD3 as a candidate lung cancer biomarker. PLOD3 gene therapy might enhance the efficacy of radiotherapy or chemotherapy in lung cancer patients.
\end{abstract}

\section{Introduction}

Lung cancer is the main cause of cancer-related morbidity, and non-small-cell lung cancer accounts for $80-85 \%$ of all lung cancer cases ${ }^{1}$. However, among these patients, only $10 \%$ achieve a complete response, and the total 5-year survival rate has remained dismal at $15 \%^{2}$ because radiation resistance severely affects the efficacy of radiotherapy ${ }^{3,4}$. Thus, we highlight the need for a greater

\footnotetext{
Correspondence: Eun Ho. Kim (eh140149@kirams.re.kr) or

S.-G. Hwang (sgh63@kcch.re.kr)

'Division of Radiation Biomedical Research, Korea Institute of Radiological \&

Medical Sciences, Seoul 01812, Korea

2Department of Molecular Cell Biology, Sungkyunkwan University School of

Medicine, Suwon 440-746, Korea

These authors contributed equally: Eun Ho Kim, Sang-Gu Hwang

Edited by G.-Q. Chen
}

understanding of the cellular and molecular targets that drive tumorigenesis to achieve better treatment efficacies.

Recently, we found four proteins, including procollagen-lysine, 2-oxoglutarate 5-dioxygenase 3 (PLOD3), which had not been previously reported to be related to radioresistance or chemoresistance ${ }^{5}$. PLOD proteins, are involved in fibrotic processes and tissue remodeling ${ }^{6,7}$. Three highly homologous PLOD isoforms have been characterized to date, including PLOD2, and PLOD3 ${ }^{8}$. PLOD3 is localized on chromosome $7 \mathrm{q} 36^{9}$, and PLOD3 activity is critical for the biosynthesis of type IV and VI collagens ${ }^{10}$. Mutations in human PLOD3 result in congenital disorders that influence the connective tissues of various organs ${ }^{11}$, suggesting that PLOD3 is crucial for normal collagen function. Collagen also is involved in

\section{(c) The Author(s) 2019}

(c) (i) Open Access This article is licensed under a Creative Commons Attribution 4.0 International License, which permits use, sharing, adaptation, distribution and reproduction c. in any medium or format, as long as you give appropriate credit to the original author(s) and the source, provide a link to the Creative Commons license, and indicate if changes were made. The images or other third party material in this article are included in the article's Creative Commons license, unless indicated otherwise in a credit line to the material. If material is not included in the article's Creative Commons license and your intended use is not permitted by statutory regulation or exceeds the permitted use, you will need to obtain permission directly from the copyright holder. To view a copy of this license, visit http://creativecommons.org/licenses/by/4.0/. 
tumor progression by modulating cancer cell migration, invasion $^{12}$, proliferation ${ }^{13}$, survival ${ }^{14}$, and metastasis ${ }^{15}$. Based on these facts, we focused on cancer cell survival with respect to PLOD3 function.

Two independent studies have reported PLOD3 mRNA overexpression in glioma and hepatocellular carcinoma tissues $^{16-18}$. PLOD3 overexpression was correlated with higher circulating protein levels in some patients ${ }^{19}$. However, the molecular mechanisms underlying the role of PLOD3 in lung cancer cell death have not been fully elucidated, and there are no data regarding the possible role of PLOD3 in lung cancer cell apoptosis. Further, the oncogenic function and prognostic value of this protein as a therapeutic and diagnostic target for lung cancer have not been revealed.

We previously found that the mechanistic target of PLOD3-induced cell death is the endoplasmic reticulum (ER)-associated stress-induced apoptosis pathway ${ }^{20,21}$, which, under physiological conditions, is activated by the accumulation of misfolded proteins in the ER to maintain cell survival ${ }^{22}$. Specifically, ER stress leads to the activation of three major unfolded protein response sensors, including pancreatic eIF2- $\alpha$ kinase (PERK), high inositolrequiring 1 (IRE1- $\alpha$ ), and ATF6. First, PERK phosphorylates the eukaryotic translation initiation factor-2a, resulting in both an initial decrease in general translation initiation and the selective translation of the transcription factor ATF6. Second, ATF6 induces growth arrest and DNA damage-inducible proteins (GADD153/CHOP), leading to cell-cycle arrest, hence preventing the damage to the cell ${ }^{23,24}$. IRE1- $\alpha$ mediates the splicing of X-boxbinding protein 1, which increases the transcription of ERresident chaperones, folding enzymes, and components of the protein degradation machinery. Third, ATF6, after activating cleavage, results in both the induction of $\mathrm{CHOP}$ and the upregulation of protein folding and degradation ${ }^{24}$. Prolonged, unresolvable ER stress overrides the salvage mechanisms of the initial unfolded protein response and eventually leads to apoptosis involving CHOP signaling, JNK activation, bcl-2 phosphorylation and depletion, and caspase cleavage (e.g., caspase-4).

Protein kinase $\mathrm{C}$ (PKC) isozymes comprise a family of at least 10 related serine-threonine kinases that play critical roles in the regulation of several cellular processes, including proliferation, cell-cycle regulation, differentiation, malignant transformation, and apoptosis ${ }^{25}$. Based on their structures and cofactor requirements, $\mathrm{PKC}$ isoforms are divided into classic PKC $(\alpha, \beta 1, \beta 2$, and $\gamma)$, novel $(\delta, \epsilon, \eta$, and $\theta)$, and atypical $(\zeta$ and $\lambda / \mathrm{i})$ groups ${ }^{25}$. Members of this family are either pro-apoptotic or anti-apoptotic, depending on the isoform and cellular context. For example, PKC $\alpha$ and PKCe inhibit apoptosis by phosphorylating or increasing the expression of the antiapoptotic protein $\mathrm{Bcl}-2$, whereas the caspase-3-dependent and caspase-2-dependent activation of $\mathrm{PKC} \delta$ promotes apoptosis via tyrosine phosphorylation, association with specific apoptotic proteins, and translocation of activated PKC $\delta$ to the mitochondria ${ }^{26}$.

Here, to develop an anti-tumor reagent, we designed human PLOD3-specific short interfering (si)RNAs to knockdown the endogenous PLOD3 overexpression in lung cancer and then investigate cell proliferation and cell death. We also assessed the possible involvement of PKC $\delta$ and PLOD3 in the ER-stress-induced cell death pathway in cell culture and in an in vivo model.

\section{Results}

\section{PLOD3 downregulation decreases radioresistance in R-H460 cells}

To determine whether PLOD3 is involved in regulating radioresistance, we tested the effects of PLOD3 depletion on cell viability and death (Fig. 1a). After siRNA transfection for $48 \mathrm{~h}$, cell viability was significantly decreased in cells transfected with PLOD3 siRNA compared to that in control siRNA-transfected cells, and this decrease was enhanced in irradiated cells (Fig. 1b, Supplementary Figure 1a). We next confirmed that PLOD3 knockdown alone or in combination with radiation led to upregulation of cleaved PARP and active-caspase-3 levels (Fig. 1c, Supplementary Figure 1b). FACS analysis showed that PLOD3 knockdown in A549 cells increased cell death $(\sim 45 \%)$ by more than 3.8 -fold compared to that in control cells $(\sim 12 \%)$, and 10-Gy radiation further increased cell death slightly (Supplementary Figure 1c, d). However, in R-H469 and A549 cells, the combination of PLOD3 knockdown and 10-Gy radiation significantly increased cell death compared to that in control cells (Fig. 1d, Supplementary Figure 1c, d). Additionally, the number of colonies formed decreased with increasing radiation doses in PLOD3 siRNA-transfected R-H460 and A549 cells, indicating a dose-dependent relationship (Fig. 1e, Supplementary Fig 1e). Therefore, our results suggest that PLOD3 depletion induces cell death to regulate radiosensitivity.

\section{PLOD3 downregulation promotes radiosensitivity and inhibits tumorigenesis in vivo}

After confirming PLOD3 transfection efficiency in vivo, we analyzed the synergistic effect of radiation and PLOD3 siRNA on R-H460 xenograft tumors. As indicated in Fig. 2a and Supplementary Fig. 2a, tumor volumes were markedly decreased by more than $\sim 36 \%$ and $47 \%$, compared to those in the control siRNA group, in the PLOD3 siRNA-treated group and radiation-treated group, respectively. The most pronounced tumor growth-inhibitory effect $(60 \%)$ was observed in the PLOD3 siRNA plus irradiation group (Fig. 2b). Further, PLOD3 protein expression was diminished in PLOD3 


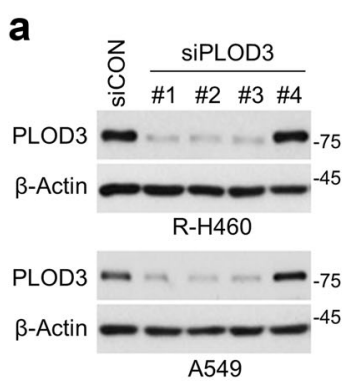

d

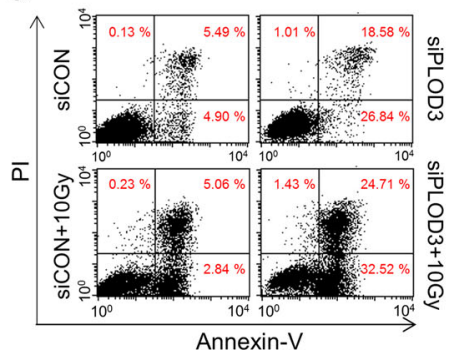

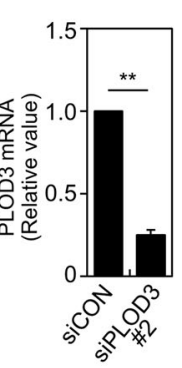

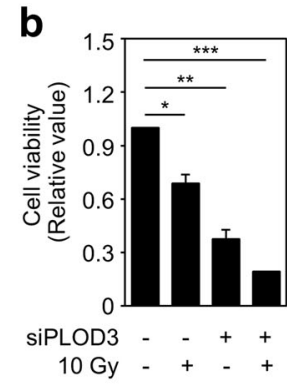

C

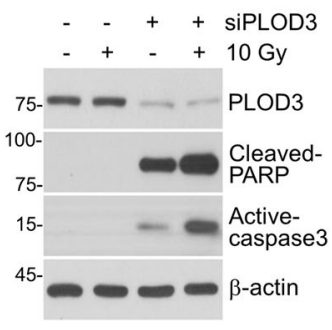

e
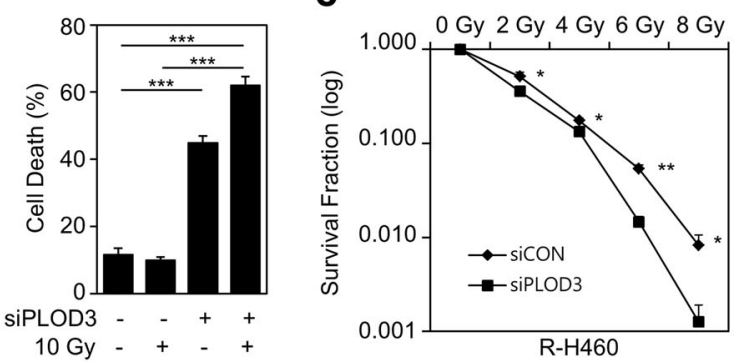

Fig. 1 Downregulation of PLOD3 decreases radioresistance in R-H460 cells. a R-H460 cells were transfected with $40 \mathrm{nM}$ control siRNA (siCON) or PLOD3 siRNA (siPLOD3) for $24 \mathrm{~h}$. Transcript and protein levels of PLOD3 were determined by western blotting (left) and qRT-PCR (right). ${ }^{* *} P<0.01$. b Analysis of the viability of cells treated with or without $10 \mathrm{~Gy}$ radiation after transfection with siCON or siPLOD3. ${ }^{*} P<0.05$; ${ }^{* *} P<0.01$; ${ }^{* * *} P<0.001$. c Protein levels of PLOD3, cleaved PARP, and active caspase-3 (cell-death marker) as determined by western blotting. $\mathbf{d}$ Determination of cell death in $\mathrm{R}-\mathrm{H} 460$ cells (treated as in $\mathbf{b}$ ) by AV/PI staining. ${ }^{* *} P<0.001$. e Surviving fraction of cells treated with a single dose of radiation ( $\left.0-8 \mathrm{~Gy}\right)$ after transfection with siCON or siPLOD3, measured two weeks after radiation treatment. ${ }^{*} P<0.05 ;{ }^{* *} P<0.01$

siRNA-injected mice compared to that in control siRNAtreated mice (Fig. 2b). Tumor weight measurements were in agreement with tumor volume results (Fig. 2c). Moreover, the PLOD3 siRNA plus radiation group showed further decreased PLOD3 protein levels compared to those in mice treated with either alone. There were possibly no visible signs of toxicity due to PLOD3 siRNA and radiation in mice, as shown by the lack of differences in body, liver, and lung weights (Fig. 2d).

Patients with high PLOD3-expressing lung cancer who were treated with radiotherapy had poorer prognosis than patients with low PLOD3-expressing lung cancer who were treated with radiotherapy in the Kaplan-Meier Plotter database (Fig. 2e). Collectively, these findings suggest that PLOD3 is correlated with tumor growth.

\section{PLOD3 knockdown induces caspase-dependent apoptosis in R-H460 cells}

To investigate the mechanisms associated with PLOD3 knockdown-induced cell death, we assessed apoptosis and necrosis in R-H460 cells by flow cytometry (Fig. 3a). At $36 \mathrm{~h}$, PLOD3 siRNA significantly increased apoptosis (AV-positive) by more than 2-fold (approximately 20\%) compared to control siRNA. Further, PLOD3 siRNAmediated apoptosis was accelerated as incubation time increased, but necrosis (AV-negative/PI-positive) was not triggered (Fig. 3b). Caspase-3 cleavage was observed at the same time point as increased apoptosis by immunoblotting analysis. Moreover, PLOD3 siRNA-induced apoptosis was caspase-dependent because Z-VAD-FMK, a pancaspase inhibitor, significantly lowered the percentage of AV-positive cells and blocked the cleavage of caspase-3 (Fig. 3c). In A549 cells, FACS and western blot analysis revealed that Z-VAD-FMK treatment decreased PLOD3 depletion-induced apoptotic cell death (Supplementary Figure $3 \mathrm{a}, \mathrm{b})$. To investigate whether cleaved caspase-3 modulates caspase activation in PLOD3-depleted cells, we measured the activity of caspase family members in RH460 cells (Fig. 3d). PLOD3 siRNA significantly increased caspase-2, 3, 4, and 6 activity by $~ 1.9-, 2.1-, 1.8-$, and 1.7fold, respectively, compared to control siRNA. Accordingly, to investigate whether caspase inhibitors could block PLOD3 knockdown-induced apoptosis (Fig. 3e), R$\mathrm{H} 460$ cells were treated with these compounds for $1 \mathrm{~h}$ and then transfected with PLOD3 siRNA. Based on AV/PI double staining, Z-VAD-FMK, Z-VDVAD-FMK, Z-DEVD-FMK, and Z-YVAD-FMK treatment reduced apoptosis by approximately $18 \%, 7 \%, 6 \%$, and $10 \%$, respectively, in PLOD3-knockdown cells. Immunoblotting results confirmed that active caspase- 3 was remarkably decreased by Z-VAD-FMK treatment, without rescuing PLOD3 protein levels, but was only slightly reduced by Z-VDVAD-FMK, Z-DEVD-FMK, and Z-YVAD-FMK. To identify additional possible 


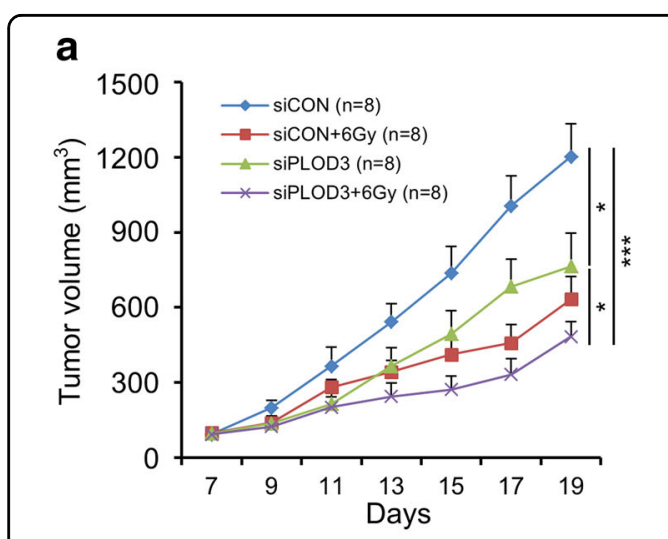

d

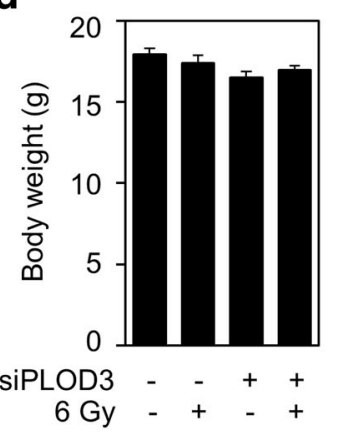

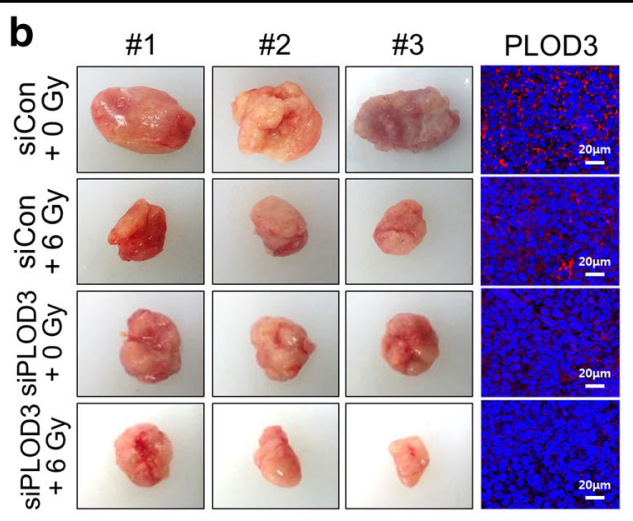

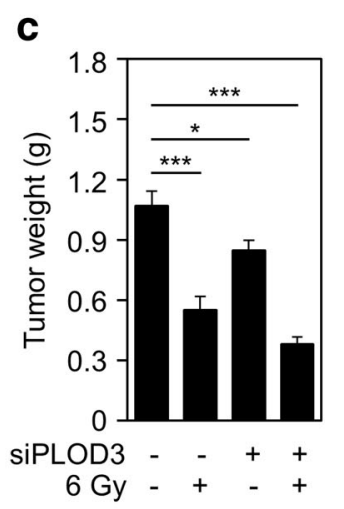

Fig. 2 Reduced PLOD3 suppresses radioresistance in $\mathbf{R}-\mathbf{H} 460$ cell xenograft tumor model. a Tumor volume was calculated at the indicated times using the formula: volume $=\left(\right.$ length $\times$ width $\left.{ }^{2} \times 3.14\right) / 6 ;(n=8)$. ${ }^{*} P<0.05 ;{ }^{* *} P<0.001$. $\mathbf{b}$ Tumors were excised and weighed at the end of the experiment (14 days). Representative images of tumors and tumor slides subjected to immunohistochemistry using an anti-PLOD3 antibody. $\mathbf{c}$ Effects of siRNA and radiation on tumor weights. Data shown are the means of each group. ${ }^{*} P<0.05$; ${ }^{* *} P<0.001$. $\mathbf{d}$ Body, liver, and lung tissues of mice were excised and weighed at the end of the experiment. All data are shown as the mean \pm SD. e Five-year survival rate curves were obtained using Kaplan-Meier Plotter (www.kmplot.com) and display the survival probability based on data of 68 lung cancer patients treated with radiotherapy

mechanisms of cellular death, we investigated autophagy and proteasome activity, which are among other mechanisms associated with anti-cancer activity. We first treated cells with PLOD3 siRNA to examine chymotrypsin-, caspase- and trypsin-like activities in R-H460 cells. Only minor changes were observed in the levels of the three proteasomal activities after PLOD3 siRNA treatment (Supplementary Figure 3c). After treatment with 3-MA, which is widely used to inhibit autophagy, RH460 cells were not significantly rescued from cell death induced by PLOD3 siRNA, and morphological changes throughout the cytoplasm and in the cell membrane were not observed (Supplementary Fig. 3d). Thus, PLOD3 ablation induces cell death that depends on apoptosis, but not autophagy and proteasome activity.

\section{PLOD3 depletion induces DNA damage and ER stress- dependent caspase activation in R-H460 cells}

Caspase- 2 has been shown to mediate DNA damageinduced apoptosis, reactive oxygen species (ROS) accumulation, and ER stress ${ }^{27}$. Caspase- 4 is also activated by ER stress $^{28}$. First, to investigate the ER localization of PLOD3 in H460 and R-H460 cells, following treatments, these organelles were visualized using Grp94 as an ER stress sensor protein, and nuclei were stained with DAPI. PLOD3 was distributed homogeneously in the cytoplasm in co-localization with Grp94 (Fig. 4a). Immunoblotting revealed that PLOD3 depletion resulted in elevated cleaved ATF6 and phosphorylation of PERK and IRE1 $\alpha$ as ER stress sensor proteins and also upregulated the DNA damage marker, $\gamma \mathrm{H} 2 \mathrm{AX}$ (Fig. 4b). qPCR confirmed increased splicing of $X B P-1$, a downstream target of IRE1 $\alpha$ (Fig. 4c). Together with ER stress and the activation of the unfolded protein response system, IRE1 $\alpha$ is reportedly involved in caspase activation. Thus, we examined whether IRE1 $\alpha$ inhibition blocked caspase activation and cell death in PLOD3-depleted cells. The IRE1 $\alpha$ inhibitor APY29 reduced PLOD3 knockdowninduced phosphorylation of IRE1 $\alpha$. APY29 also reduced cleaved caspase- 2 and procaspase- 4 and the proportion of apoptotic cells (Fig. 4d). R-H460 cells were treated with combinations of PLOD3, IRE1 $\alpha$, and control siRNA for $48 \mathrm{~h}$ and apoptosis was analyzed by AV/PI staining (Fig. 4e). PLOD3 siRNA led to an increase in the apoptotic cell population $(\sim 32 \%)$; however, combined transfection with IRE1 $\alpha$ siRNA significantly reduced this by more than 


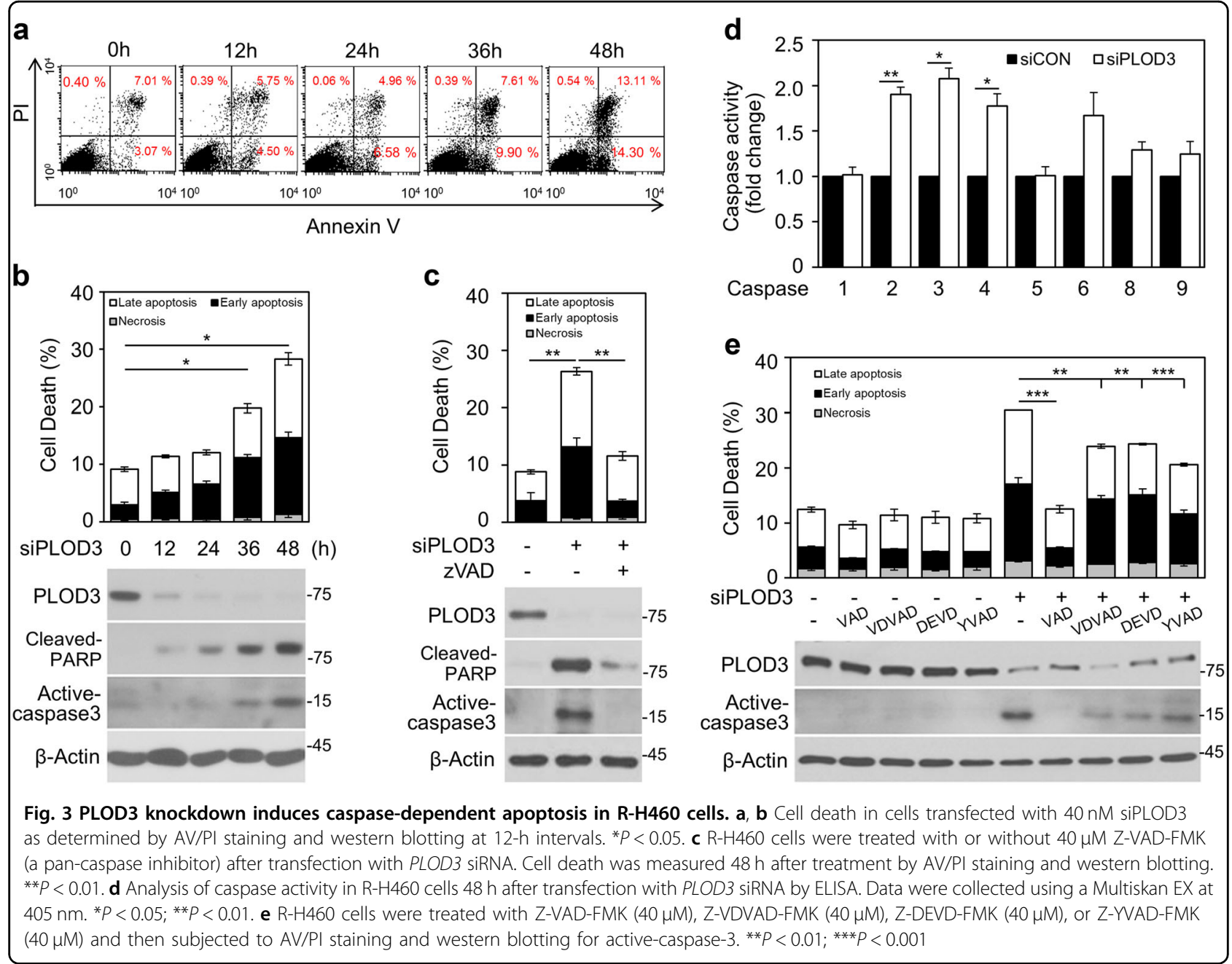

8\% compared to PLOD3 siRNA alone. Notably, we found that caspase inhibition in PLOD3-knockdown R-H460 cells did not regulate ER stress (Fig. 4f). Furthermore, caspase inhibition using Z-VAD-FMK completely inhibited $\gamma \mathrm{H} 2 \mathrm{AX}$ accumulation after PLOD3 knockdown (Fig. 4f). These results suggested that DNA damage is induced by caspase activity after PLOD3 depletion. However, PLOD3 knockdown-induced cell death was not found to depend on ROS levels (Fig. 4g). These results suggest that caspase- 2 and 4 depend on IRE1 $\alpha$ activation in PLOD3 siRNA-treated cells.

\section{PLOD3 regulates PKC-dependent apoptosis by activating $\mathrm{PKC} a$ and $\mathrm{PKC} \delta$ via direct interactions}

It is well-known that $\mathrm{PKC} \alpha$ or $\mathrm{PKC} \delta$ is involved in the ER stress response, leading to apoptosis ${ }^{29,30}$. Moreover, PKC $\delta$ reportedly associates with and phosphorylates caspase-3 to promote apoptotic activity, after which activated caspase- 3 cleaves PKC $\delta^{31}$. Thus, to investigate the relationship between PLOD3 and PKC, we compared the subcellular localization of these markers in
R-H460 cells (Fig. 5a). The data showed that PLOD3 co-localized with PKC $\alpha$ and $\mathrm{PKC} \delta$ in the nucleus and cytosol. Interestingly, PKC $\delta$ localized to the ER, similar to PLOD3. Next, as PLOD3 co-localized with PKCs, we evaluated the associated interactions in R-H460 cells. We immunoprecipitated exogenous PLOD3 from HA-PLOD3-, PKC $\alpha-$, and GFP-PKC $\delta$-transfected R-H460 cell lysates using a HA antibody and detected the immune complexes with HA, PKC $\alpha$, and GFP antibodies (Fig. 5b, c). We observed an interaction between PLOD3 and PKCs in R-H460 cells. A proximity ligation assay confirmed that endogenous PLOD3 interacts with PKCs in vivo(Fig. 5 d). A proximity ligation assay probe was used as a negative control. To examine whether PKC activity changes in the absence or presence of PLOD3, we transfected R-H460 cells with PLOD3 siRNA. PKC $\alpha$ and PKC $\delta$ phosphorylation was remarkably increased by PLOD3 knockdown in R-H460 cells (Fig. 5e). These results suggest that PLOD3 regulates the activity of $\mathrm{PKC} \alpha$ and $\mathrm{PKC} \delta$, leading to PKC-dependent apoptosis. Furthermore, we examined the localization of 


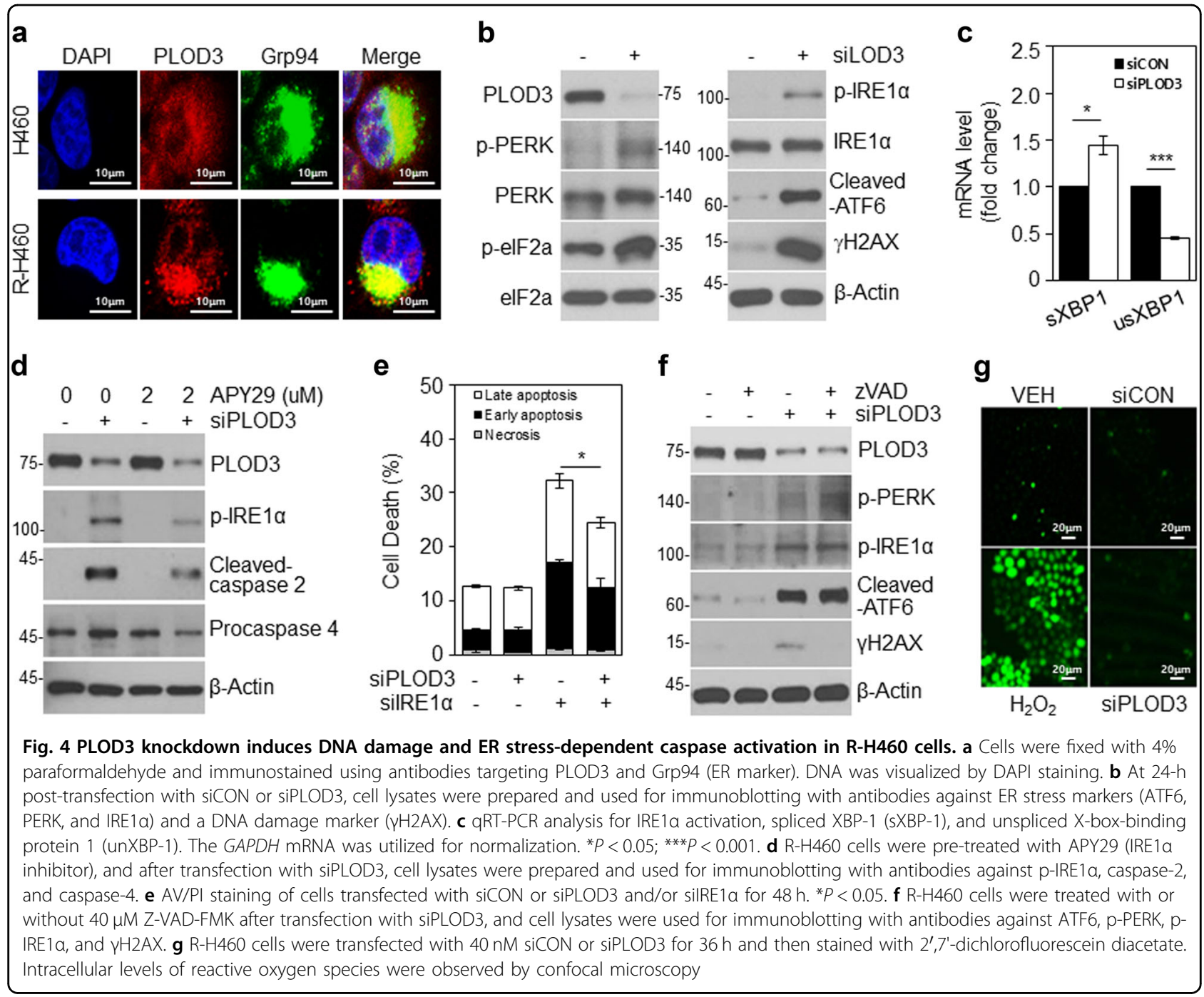

cleaved $\mathrm{PKC} \delta$, which is important for PKC $\delta$-dependent apoptosis. Cleaved PKC $\delta$ was significantly induced in PLOD3-knockdown cells and was localized to the nucleus (Fig. 5f). Thus, we hypothesized that PKCs are related to PLOD3 depletion mediated-apoptosis in R$\mathrm{H} 460$ cells. Accordingly, we investigated whether $P K C \delta$ siRNA blocks PLOD3 knockdown-induced subcellular events. R-H460 cells were treated with combinations of $P L O D 3, P K C \delta$, and control siRNA for $48 \mathrm{~h}$ and apoptosis was analyzed by AV/PI staining (Fig. 5g). PLOD3 siRNA led to an increase in the apoptotic cell population ( 39\%); however, combined transfection with $P K C \delta$ siRNA significantly suppressed this increase by more than 14\%. Immunoblotting results confirmed that cleaved PARP and active caspase-3 were remarkably decreased, without rescuing PLOD3 protein levels (Fig. 5g). Based on the results of interaction with PLOD3 and $\mathrm{PKC} \alpha$ and increased PKC $\alpha$ phosphorylation by
PLOD3 depletion, we examined whether $P K C \alpha$ siRNA blocks PLOD3 knockdown-induced apoptosis. To this end, R-H460 cells were treated with a combination of PLOD3, $P K C \alpha$, and control siRNA for $48 \mathrm{~h}$. Apoptosis was then analyzed by AV/PI staining and immunoblotting (Fig. 5h). PLOD3 siRNA increased the apoptotic population ( $41 \%)$, but combined transfection with $P K C \alpha$ siRNA suppressed this increase by more than $13 \%$. Immunoblotting data confirmed that cleaved PARP and active caspase- 3 were remarkably decreased, without rescuing PLOD3 protein level. Further, IRE1 $\alpha$ and elF2a phosphorylation, as assessed by immunological analysis, was similar to that of cell death markers (Fig. 5i). Therefore, PKC $\delta$ siRNA inhibits the PLOD3induced ER stress response. Next, we examined whether combined PLOD3 and PKC $\delta$ siRNAs could affect caspase activity (Fig. 5j). PLOD3 siRNA significantly increased caspase- 2 activity by approximately 1.6 -fold 


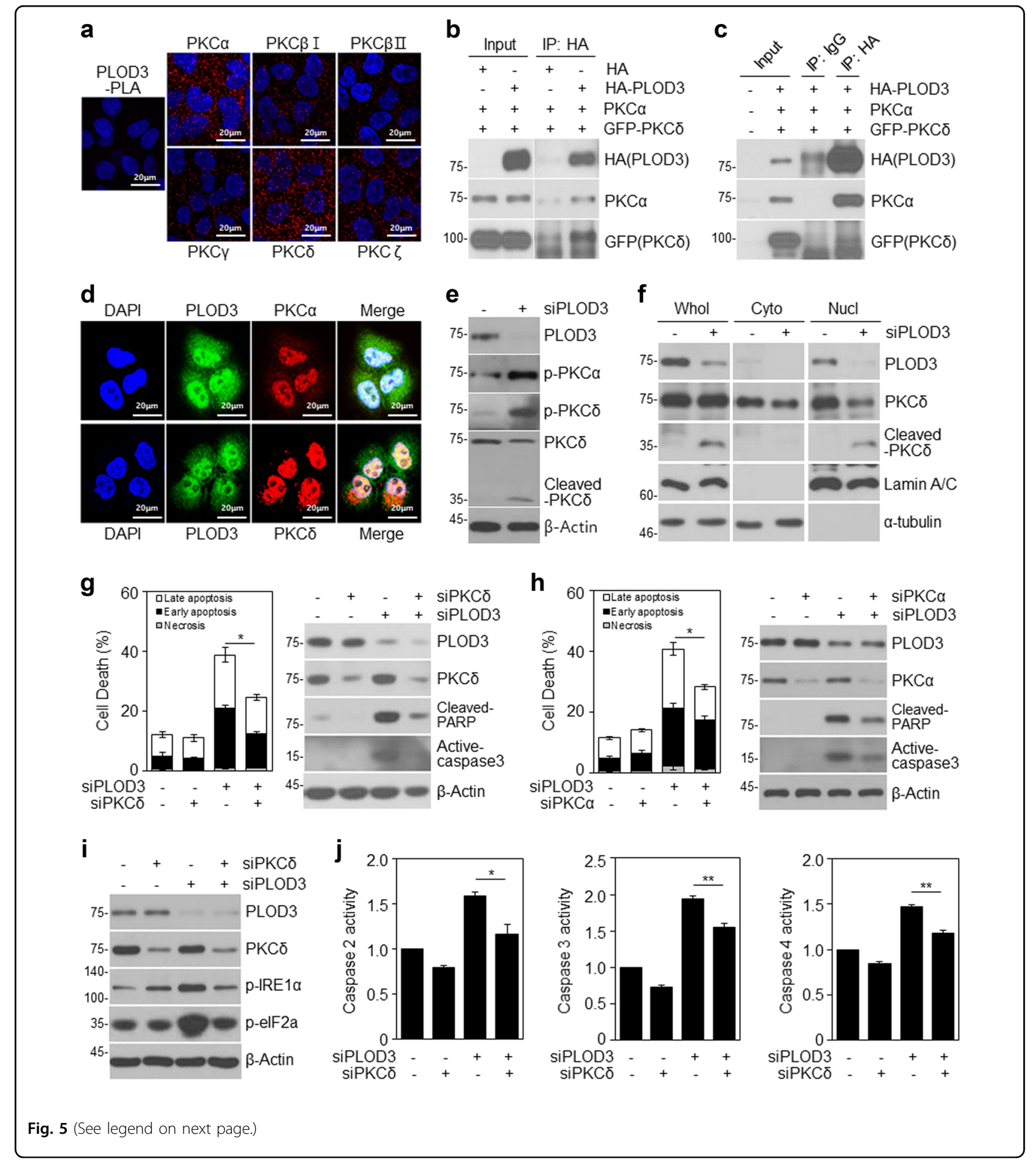

compared to control siRNA, but combined transfection with $P K C \delta$ siRNA further enhanced this increase by 1.2-fold. Further, PLOD3 siRNA significantly increased caspase-3 activity by $\sim 2$-fold compared to that with control siRNA, but combined transfection with $P K C \delta$
siRNA enhanced this increase by $\sim 1.5$-fold. Moreover, PLOD3 siRNA significantly increased caspase-2 activity by $\sim 1.5$-fold compared to control siRNA, but combined transfection with $P K C \delta$ siRNA enhanced this increase by $\sim 1.2$-fold. Taken together, these results suggest that 
(see figure on previous page)

Fig. 5 PLOD3 knockdown induces activation of PKCs and PLOD3 directly interacts with PKCs. a R-H460 cells were fixed and incubated with a mouse anti-PLOD3 antibody together with rabbit antibodies against PKCs, followed by in situ proximity ligation assay analysis. Representative confocal images of cells with proximity ligation assay-positive signals (red dots). b, c R-H460 cells were transfected with HA-PLOD3, PKCa, or GFPPKC . Cell lysates were immunoprecipitated with normal lgG (a negative control) or HA antibody, and then immunocomplexes were resolved by SDS-PAGE and immunoblotted with antibodies against HA, PKCa, and GFP. $\mathbf{d}$ Immunostaining analysis of the localization of PKCs in R-H460 cells. Cells were fixed with 4\% paraformaldehyde and immunostained for PLOD3 (green), PKCa (red), and PKC $\delta$ (red). DNA was visualized by DAPI staining. e PLOD3 or control siRNA was transfected into R-H460 cells; 24-h post-transfection, cell lysates were prepared and used in immunoblotting with antibodies targeting PLOD3, p-PKCa, PKC, p-PKC $\delta$, and $\beta$-actin. f Subcellular fraction analysis of R-H460 cells following siCON or siPLOD3 treatment. Proteins in each fraction were resolved by SDS-PAGE and immunoblotted with antibodies against PLOD3, PKC $\delta$, lamin A/C, and a-tubulin. g R-H460 cells were transfected with $40 \mathrm{nM}$ siCON or siPLOD3 and/or siPKC $\delta$ for $48 \mathrm{~h}$. Cell death in R-H460 cells was determined by AV/PI staining (left). Protein levels of the indicated proteins were determined by western blotting (right). ${ }^{*} P<0.05$. $\mathbf{h}$ R-H460 cells were transfected with $40 \mathrm{nM}$ siCON or siPLOD3 and/or siPKCa for $48 \mathrm{~h}$. Cell death in R-H460 cells was determined by AV/PI staining (left). Protein levels of the indicated proteins were determined by western blotting (right). ${ }^{*} P<0.05$. i Protein levels of PLOD3, PKC , p-elF2a, and p-IRE1 a were determined by western blotting. $\mathbf{j}$ Caspase-2, caspase-3, and caspase- 4 activities in R-H460 cells (treated as in $\mathbf{b}$ ) were determined by caspase activity assay. Data were collected using the Multiskan EX at $405 \mathrm{~nm}$. ${ }^{*} P<0.05 ;{ }^{*} P<0.01$

PKCס, downstream of PLOD3, plays an important role in the PLOD3-mediated cell death mechanism.

\section{Loss of PLOD3 overcomes chemoresistance in vitro}

Next, we examined whether PLOD3 status influences the chemosensitivity of cancer cells. Interestingly, radioresistant R-H460 cells were more resistant to cisplatin than their parental $\mathrm{H} 460$ cells, and resistance levels were similar to those of A549 cells (Fig. 6a, c). Next, we investigated the combined effect of cisplatin and PLOD3 siRNA on the viability and cell death of R-H460 and A549 cells. The combinatorial treatment attenuated cell viability and increased cell death compared to cisplatin alone (Fig. 6b, d), suggesting that PLOD3 status might influence the chemosensitivity of cancer cells to cisplatin. Next, to investigate the effect of PLOD3 knockdown on apoptosis, we measured the expression of cleaved PARP and active caspase- 3 by western blot analyzes (Fig. 6e). Compared to cisplatin alone, the combination of PLOD3 siRNA and cisplatin resulted in significant increases in the levels of these two proteins in $\mathrm{R}-\mathrm{H} 460$ and A549 cells. Whereas transfection of R-H460 cells with PLOD3 siRNA alone induced approximately $47 \%$ cell death compared to $10 \%$ in controls, the co-stimulation of PLOD3 siRNAtransfected cells with etoposide, hydroxyurea, or doxorubicin induced approximately $72 \%, 70 \%$, and $65 \%$ cell death, respectively (Fig. 6f). Taken together, these results suggest that PLOD3 status is strongly associated with cancer cell chemosensitivity.

\section{Discussion}

PLOD3, which we identified as a radioresistance-related protein, has already been highlighted for its potential role in hepatocellular carcinoma, glioma, skin cancer, and prostate cancer ${ }^{16-19}$. However, for lung cancer, the specific mechanism of PLOD3 regulation in cell death has not been elucidated.
In the current study, PLOD3 siRNA inhibited the proliferation of lung cancer cells and induced cell death, an effect that was augmented by radiation treatment, in cells and an in vivo model. We also found that (a) PLOD3 is differentially expressed during tumor progression, $(b)$ PLOD3 expression promotes apoptosis in lung cancer by activating caspase-2,4-dependent signaling through ER stress-induced IRE1 $\alpha$ activation and caspase-3-dependent apoptosis, and (c) PLOD3 siRNA, combined with chemotherapeutic drugs, can increase cancer cell death, suggesting that PLOD3 is a potential therapeutic target in cancer. Thus, the present study provided the first evidence that PLOD3 inhibition can induce apoptosis in lung cancer cells, in addition to helping overcome radioresistance or chemoresistance.

Currently, PLOD2 is reportedly induced by hypoxiainducible factor- $1 \alpha$ under hypoxic conditions, which in turn enhances hypoxia-induced epithelial-mesenchymal transition in glioma ${ }^{32}$ and breast cancer cells ${ }^{33}$. Hypoxiainducible factor- $1 \alpha$ also regulates PLOD1 transcription in breast cancer; however, PLOD2 activity is more critical for HIF-1-induced cancer progression ${ }^{33}$. PLOD2 is also directly regulated by miR-26a-5p and miR-26b-5p, and PLOD2 expression is reportedly a possible prognostic marker for patients with bladder cancer ${ }^{34}$ and renal cell carcinoma $^{35}$. Moreover, the E2Fs ${ }^{36}$ and FOXA1 ${ }^{37}$ transcription factors have been demonstrated as regulators of PLOD2 during cancer progression. However, the regulation of PLOD1 and PLOD3 expression is not well understood. Thus, the upstream regulation of PLOD3 that could lead to the induction of cell death in our system remains to be elucidated. One report showed that miR-663a reduces PLOD3 biosynthesis by targeting the 3 '-untranslated region of PLOD3 mRNA, suggesting the interrelationship of this microRNA in regulating collagen IV secretion under physiological conditions and in response to ER stress. Consistent herewith, we focused on 

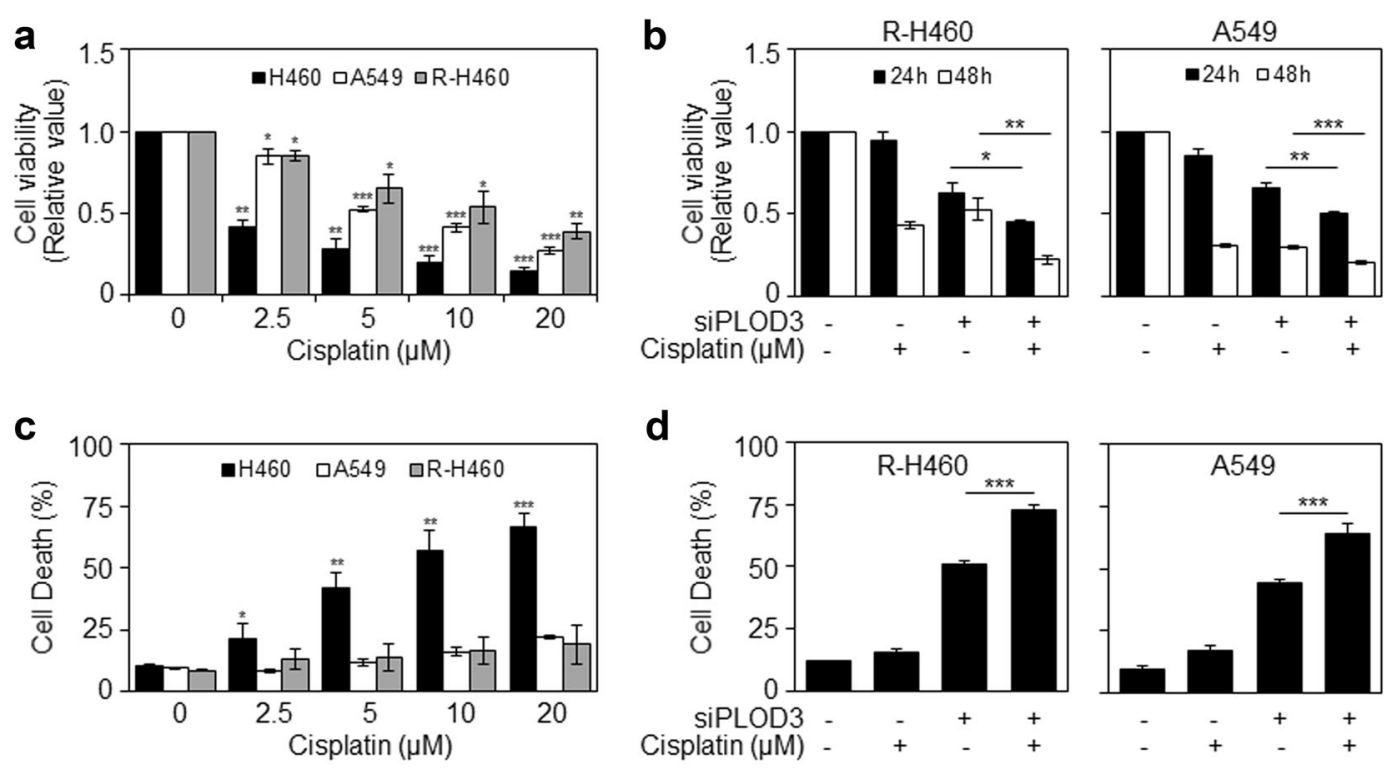

d
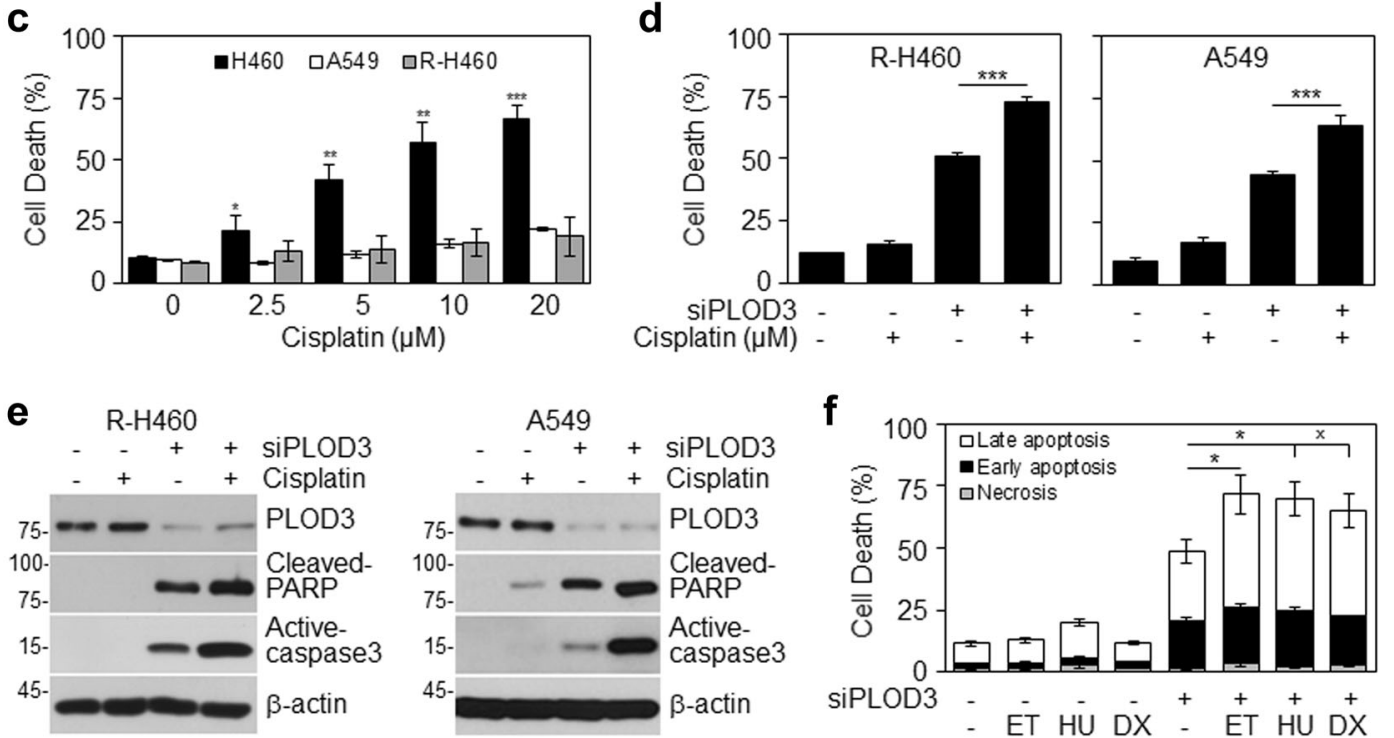

Fig. 6 PLOD3 depletion overcomes chemoresistance in vitro. a Cells were treated with cisplatin for 48 h. Proliferation was detected by cell viability assays. ${ }^{*} P<0.05 ;{ }^{*} P<0.01 ;{ }^{* *} P<0.001$. b A549 and R-H460 cells were transfected with $40 \mathrm{nM}$ siCON or siPLOD3 for $24 \mathrm{~h}$ and then treated with cisplatin for another $48 \mathrm{~h}$. The proliferation rate was determined by cell viability assays. ${ }^{*} P<0.05 ;{ }^{* *} P<0.01$; ${ }^{* * *} P<0.001$. c Cisplatin-treated cells (for $48 \mathrm{~h}$ ) were analyzed by FACS after AV/PI staining. ${ }^{*} P<0.05$; ${ }^{*} P<0.01$; ${ }^{* *} P<0.001$. $\mathbf{d}$ Cell death (of cells treated as in b) as analyzed by AV/PI staining. ${ }^{* *} P<0.001$. e Protein levels of cleaved-PARP and active-caspase-3 as determined by western blotting after PLOD3 siRNA and cisplatin treatment. $\mathbf{f}$ R-H460 cells were transfected with siRNA in the absence or presence of $5 \mu \mathrm{M}$ etoposide (ET) or $1 \mathrm{mM}$ hydroxyurea (HU) or $0.1 \mu \mathrm{g} / \mathrm{ml}$ doxorubicin (DX). At $48 \mathrm{~h}$ after the drug treatments, the cells were analyzed by FACS after AV/PI staining. ${ }^{*} P<0.05$

ER stress as an apoptosis-inducing factor as collagen is a major secretory protein in a variety of tissues, and defective secretion could result in physiological ER stress, fibrosis, and a number of disease states. As the pathway identified herein might represent a major physiological mechanism in lung cancer, it will be crucial to establish the role of the PKC 8 -PLOD3-collagen axis in different tissues in future. Moreover, as PLOD3 is also present in the extracellular space, as well as in the serum and on the cellular surface ${ }^{19}$, our results suggest that $\mathrm{PKC} \delta$ as a downstream effector of PLOD3 might also modulate other important extracellular PLOD3 functions ${ }^{38}$.

PLOD3 mutations are reportedly associated with connective tissue disorders ${ }^{11}$. PLOD3 knockout in embryos and cells was reported to be associated with reduced glycosylated hydroxylysines on type IV and VI collagen and abnormal distribution ${ }^{39}$. PLOD3 is overexpressed in hepatocellular carcinoma ${ }^{40}$ and is a potential diagnostic marker for early-stage disease ${ }^{40}$. Further, PLOD3 knockdown controls liver tumor incidence and growth rates in a spontaneous mouse model of hepatocellular carcinoma ${ }^{40}$. Our study showed consistent results for lung cancer and suggests that secreted PLOD3 could serve as a potential inducer of lung cancer metastasis or as a prognostic marker. We are currently expanding this mechanistic investigation in relation to metastasis using lung cancer cells and patient samples.

Interestingly, as we observed nuclear localization of cleaved PKC $\delta$ following PLOD3 knockdown, we hypothesized that PLOD3 siRNA, via the production of ROS, might induce post-translational modifications in $\mathrm{PKC} \delta$, which allow its translocation to the nucleus. However, these events did not depend on ROS levels. Moreover, the hinge domain of PKC $\delta$ is the site for caspase-3 cleavage, 
which happens in the nucleus and laeds to the release of the $\delta$-catalytic fragment $(\delta \mathrm{CF})$, corresponding to the kinase domain. Thus, in some systems, apoptotic cell death is associated with caspase 3-dependent PKC $\delta$ cleavage; the formation of the catalytically active fragment of PKC $\delta$ in our cellular model following PLOD3 siRNA treatment should be explored further in future studies.

Recently, diverse molecular biomarkers of drug resistance have been identified. For example, the protein disulfide isomerases PDIA4 and PDIA6 regulate resistance to cisplatin-induced apoptosis in lung adenocarcinoma ${ }^{41}$. The copper transporter CTR1 is involved in the uptake of cisplatin and has been developed as a therapeutic target ${ }^{42}$. Thus, medical protocols should be planed according to the character of each patient and it needs to find more efficient biomarkers for identifying the molecular mechanisms of cisplatin resistance. Our results suggest that chemotherapy could be more effective in combination with RNAi-mediated knockdown of PLOD3. Certainly, for the improvement of such a therapeutic strategy for clinical application, a suitable vector system is necessary. We also need to explore the effects of PLOD3 knockdown on normal tissues in future before clinical application of this strategy can be contemplated. We demonstrated that the level of activated PKC $\delta$ or PKC $\alpha$ was increased upon treatment with siPLOD3 and that the siPLOD3-induced apoptosis was decreased by inhibiting the expression of PLOD3 and PKC $\delta$ or PKC $\alpha$. Thus, we are further planning to identify the functional relationship between PLOD3 and PKC $\delta$ or PKC $\alpha$; the cellular events or functional mechanisms underlying these also need to be elucidated.

In summary, the PLOD3 siRNA used in this study are potent tools to modulate PLOD3 expression and they might ultimately be developed into attractive anti-tumor therapeutics. Further, PLOD3 might represent a prognostic biomarker and a target for reversing cisplatin resistance in lung cancer.

\section{Materials and methods}

\section{Cell culture and treatment}

Human lung cancer cell lines (H460 and A549) were purchased from ATCC (Manassas, Virginia, USA), and we established a radioresistant $\mathrm{H} 460$ (R-H460) cell line derived from parental radiosensitive $\mathrm{H} 460$ lung cancer cells treated cumulatively with $2 \mathrm{~Gy}$ radiation twice a week for 20 weeks $^{5}$. H460, A549, and R-H460 cells were cultured in RPMI-1640 medium supplemented with $10 \%$ fetal bovine serum. Cells were irradiated using a $137 \mathrm{Cs}-$ ray source (Atomic Energy of Canada) at a dose rate of $3.81 \mathrm{~Gy} / \mathrm{min}$. Where indicated, cells were treated with etoposide, hydroxyurea, doxorubicin, and cisplatin to induce apoptosis. Caspase inhibitors [Z-VAD-FMK (Adipogen, Z-VDVAD-FMK, Z-DEVD-FMK, and Z-
YVAD-FMK (R\&D Systems)] were used to block caspase activation. Inhibitor APY29 was used to block IRE1 $\alpha$ activation. Where indicated, cells were treated with 3methyladenine to block autophagy.

\section{PLOD3 siRNA transfections}

The following human PLOD3-specific siRNAs, synthesized by Genolution, were used: \#1; 5'-GGUUAAAGA AGGAAAUGGAUU-3', \#2; 5'-GGAAGUACAAGG AUGAUGAUGACGACGA-3', \#3; 5'-AUAUGAUCAUC AUGUUUGUUU-3', \#4; 5'-GCCUUAAUCUGGAUCA UAAUU-3'. The siRNA duplexes were transfected into cells using Lipofectamine ${ }^{\circledast}$ RAiMAX Reagent according to the manufacturer's guidelines.

\section{Western blot analysis}

Western blot analyzes were performed as described previously ${ }^{16}$ using primary antibodies targeting the following proteins: PLOD3 (Proteintech Group, Chicago, IL, USA); p-PERK, PERK, p-eIF2 $\alpha$, HA, GFP, lamin A/C, $\alpha$ tubulin, PKC $\alpha, \quad P K C \delta$ (Santa Cruz Biotechnology); cleaved-PARP (Asp214), cleaved caspase-3, eIF2 $\alpha$, IRE1 $\alpha$, $\gamma \mathrm{H} 2 \mathrm{AX}$, and $\mathrm{p}-\mathrm{PKC} \delta$ (Cell Signaling Technology); $\mathrm{p}-$ IRE1 $\alpha$ (Novous); ATF6, caspase- 2 and caspase-4 (Abcam); and $\beta$-actin (Sigma) was used as a loading control.

\section{Quantitative reverse transcription-polymerase chain reaction (qRT-PCR)}

Total RNA was isolated using an RNeasy Mini kit (Qiagen). qPCR was performed in triplicate using a PIKOREAL 96 (Thermo) and SYBR Premix Ex Taq (Takara Bio, Shiga, Japan). A two-temperature thermocycling program was used, with 42 cycles of $95^{\circ} \mathrm{C}$ (denaturation) and $55^{\circ} \mathrm{C}$ (annealing). Target-gene amplification signal was normalized to that of GAPDH in the same reaction.

\section{Cell viability assay}

Cells were seeded at 5000 cells/well in a 96-well plate and incubated for $24 \mathrm{~h}$ in accordance with the indicated experimental conditions. For quantification of cell viability, an equal volume of culture medium containing Cyto $\mathrm{X}^{\mathrm{TM}}$ Reagent (LPS solution) was added to the cells and the plate was incubated for $4 \mathrm{~h}$. Cell viability was then measured using Multiskan EX (Thermo Fisher Scientific, Germany) at $450 \mathrm{~nm}$.

\section{Fluorescence-activated cell sorting (FACS)}

Cells $\left(3 \times 10^{5}\right)$ were seeded in a $60-\mathrm{mm}$ dish and exposed to the indicated experimental conditions. For quantification of cell death, cells were trypsinized, washed in phosphate-buffered saline (PBS), and then resuspended in $1 \times$ binding buffer. Cells were incubated with annexin $\mathrm{V}(\mathrm{AV})$ and propidium iodide (PI) for 
$15 \mathrm{~min}$, and analyzed with a FACScan flow cytometer (Becton Dickinson).

\section{Clonogenic assay}

Cells were seeded in triplicate into $60-\mathrm{mm}$ tissue culture dishes at $2,4,8,16$, and $32 \times 10^{2}$ cells/dish and exposed to $0,2,4,6$, and $8 \mathrm{~Gy}$, respectively. Cells were exposed once to different doses of radiation. After 14 days, colonies arising from surviving cells were stained with trypan blue and counted using a colony counter (Imaging Products).

\section{Tumor xenograft animal model}

Studies with the tumor xenograft animal model were performed as described previously ${ }^{43}$ using control siRNA or PLOD3 siRNA \#2 (KIRAMS 2015-0070).

\section{Immunofluorescence confocal microscopy}

Immunofluorescence staining was performed as described previously ${ }^{43}$ using primary antibodies against PLOD3 (Proteintech), PKC $\alpha$, PKC 8 , and GRP94 (Santa Cruz Biotechnology). Cell nuclei were counter-stained with DAPI. Images were acquired using a confocal laserscanning microscope (model LSM 710, Carl Zeiss) and were processed with ZEN 2009 Light Edition (Carl Zeiss).

\section{Data mining using Kaplan-Meier plotter}

Kaplan-Meier survival curves in relation to PLOD3 expression were generated for lung cancer, using KM Plotter (http://kmplot.com/). Cancer type was defined as lung cancer and data type as mRNA, whereas analysis type was defined as cancer vs. normal.

\section{Caspase activity assay}

Caspase activities were measured using caspase family activity assay kits (Abcam) according to the manufacturer's recommendations. Data were collected using a Multiskan EX at $405 \mathrm{~nm}$.

\section{Proteasome activity}

Proteasome activities were measured using proteasome$\mathrm{Glo}^{\mathrm{TM}}$ chymotrypsin-like, trypsin-like, and caspase-like cell-based assays (Promega) according to the manufacturer's recommendations. Data were collected using a Victor X2 multi-label reader (Perkin Elmer).

\section{ROS assay}

R-H460 cells were treated with siRNA under the indicated experimental conditions. The cells were incubated

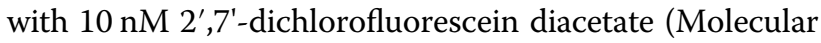
Probes) in the dark at $37^{\circ} \mathrm{C}$ for $30 \mathrm{~min}$. Cell staining was examined with a laser-scanning confocal microscope (model LSM 710, Carl Zeiss) equipped with an argon laser tuned to an excitation wavelength of $488 \mathrm{~nm}$, LP505 emission filter (515-540 nm), and Zeiss Axiovert -100X objective lens. Two groups of cells were randomly selected from each sample.

\section{In situ proximity ligation assay}

Paraformaldehyde-fixed R-H460 cells were permeabilized with $0.2 \%$ Triton X-100, washed, and blocked with blocking solution (Olink Bioscience). Mouse monoclonal anti-PLOD3 antibody (Proteintech Group) together with rabbit polyclonal antibodies against $\mathrm{PKC} \alpha, \mathrm{PKC} \beta \mathrm{I}$, PKC $\beta I I, P K C \gamma, P K C \delta$, and PKC $\zeta$ (Santa Cruz Biotechnology) were used for the proximity ligation reaction. The assay was conducted using the Duolink Detection Kit (Sigma) according to the manufacturer's protocol.

\section{Plasmid construction and transfection}

Plasmids were constructed by standard cloning techniques and were verified by DNA sequencing. Human PLOD3 cDNA (wild type) purchased from Origene (Cat. No. SC324563) was PCR-amplified and cloned into the pcDNA3.1 vector. Wild type PLOD3 was amplified with primers 5'-GATGGATCCATGACCTCCTCGGGGCCT GGA-3' (BamHI) and 5'-TCTCGAGTCAGGGGTCGAC AAAGGAC-3' (Xhol), and the amplicon was inserted between BamHI and XhoI in the pHA vector. PKC $\alpha$ and GFP-PKC $\delta$ expression vectors were purchased from the Korea Human Gene Bank. Plasmids were transfected using Mirus-2020 Reagent according to the manufacturer's guidelines.

\section{Immunoprecipitation}

R-H460 cells were transfected with vectors under the indicated experimental conditions for $48 \mathrm{~h}$. The cells were washed twice with PBS, harvested, and lysed for $30 \mathrm{~min}$ in NP-40 buffer [50 mM Tris- $\mathrm{HCl}$ (pH 8), $150 \mathrm{mM} \mathrm{NaCl}, 1 \%$ NP-40, and 100X protease and phosphatase inhibitor cocktail]. Samples were diluted to $500 \mu \mathrm{g}$ of protein in $800 \mu \mathrm{l}$ of buffer and pre-cleared for $1 \mathrm{~h}$ at $4{ }^{\circ} \mathrm{C}$ with $50 \mu \mathrm{l}$ of a $50 \%$ slurry of protein A/G-Sepharose beads (GE Healthcare). After brief centrifugation to remove precleared beads, $1 \mu \mathrm{g}$ of antibody against HA (Santa Cruz Biotechnology) was added to each sample and incubated on a rocking platform at $4{ }^{\circ} \mathrm{C}$ overnight. The immune complex was precipitated by incubation with $40 \mu \mathrm{l}$ of protein A/G-Sepharose beads at $4{ }^{\circ} \mathrm{C}$ for $4 \mathrm{~h}$. The beads were washed thrice with immunoprecipitation buffer and then boiled with sample buffer $[0.1 \mathrm{M}$ Tris- $\mathrm{HCl}(\mathrm{pH} 6.8)$, $4 \%$ SDS, $40 \mathrm{mM}$ EDTA, $20 \%$ glycerol, and $\beta$ mercaptoethanol].

\section{Subcellular fractionation}

Cells were lysed using subcellular fractionation buffer (250 mM sucrose, $20 \mathrm{mM}$ HEPES (pH 7.4), $10 \mathrm{mM} \mathrm{KCl,}$ $1.5 \mathrm{mM} \mathrm{MgCl} 2,1 \mathrm{mM}$ EDTA, $1 \mathrm{mM}$ EGTA, $1 \mathrm{mM}$ DTT, and protease inhibitors), and the lysate was passed 
through a 25 -gauge needle 10 times using a 1-ml syringe. The lysate was placed on ice for $20 \mathrm{~min}$ and centrifuged at $3000 \mathrm{rpm}$ for $5 \mathrm{~min}$ to obtain nuclear pellet. The pellet was washed with fractionation buffer 11 times, and the supernatant was centrifuged at $8000 \mathrm{rpm}$. The supernatant here refers to the cytosolic and membrane fraction.

\section{Statistical analysis}

Cell culture experiments were performed at least in triplicate. All data are expressed as mean \pm standard deviation values. Statistical differences between groups were assessed using Student's $t$-test (two-tailed) analysis. $P$-values were interpreted as follows: not significant (n.s.), ${ }^{*} P<0.05,{ }^{* *} P<0.01$, and ${ }^{* * *} P<0.001$.

\section{Acknowledgements}

This work was supported by a National Research Foundation of Korea (NRF) grant (No. 2017 M2A2A7A01019417) and a grant from the Korea Institute of Radiological and Medical Sciences (KIRAMS), which was funded by the Ministry of Science, ICT (MSIP) Republic of Korea (50531-2018).

\section{Author contributions}

S.H. and E.K. conceived the idea, designed the experiment, and wrote the manuscript. J.B., H.Y., G.K., J.L., J.K., Y.J., J.C., C.L., J.S., J.A., and J.K. performed the experiments. S.H. and E.K analyzed the data. All authors read and approved the final version of the manuscript.

\section{Conflict of interest}

The authors declare that they have no conflict of interest.

\section{Publisher's note}

Springer Nature remains neutral with regard to jurisdictional claims in published maps and institutional affiliations.

Supplementary Information accompanies this paper at (https://doi.org/ 10.1038/541419-019-1405-8).

Received: 30 August 2018 Revised: 28 January 2019 Accepted: 30 January 2019

Published online: 15 February 2019

\section{References}

1. Rebecca, S., Deepa, N. \& Ahmedin, J. Cancer statistics, 2012. CA Cancer J. Clin. 62, 10-29 (2012).

2. Jemal, A. et al. Global cancer statistics. CA Cancer J. Clin. 61, 69-90 (2011).

3. Begg, A. C., Stewart, F. A. \& Vens, C. Strategies to improve radiotherapy with targeted drugs. Nat. Rev. Cancer 11, 239 (2011).

4. Ogawa, K. et al. Radiotherapy targeting cancer stem cells: current views and future perspectives. Anticancer Res. 33, 747-754 (2013).

5. Yun, H. S. et al. Radiotherapy diagnostic biomarkers in radioresistant human H460 lung cancer stem-like cells. Cancer Biol. Ther. 17, 208-218 (2016).

6. Hausmann, E. Cofactor requirements for the enzymatic hydroxylation of lysine in a polypeptide precursor of collagen. Biochimica et Biophysica Acta (BBA)Protein. Structure 133, 591-593 (1967).

7. Rhoads, R. E. \& Udenfriend, S. Decarboxylation of alpha-ketoglutarate coupled to collagen proline hydroxylase. Proc. Natl. Acad. Sci. USA 60, 1473-1478 (1968).

8. Salo, A. M. et al. The lysyl hydroxylase isoforms are widely expressed during mouse embryogenesis, but obtain tissue-and cell-specific patterns in the adult. Matrix Biol. 25, 475-483 (2006).

9. Hautala, T. et al. Cloning of human lysyl hydroxylase: Complete cDNA-derived amino acid sequence and assignment of the gene (PLOD) to chromosome 1p36. 3KI, MyllylGenomics 13, 62-69 (1992).
10. Rautavuoma, K. et al. Premature aggregation of type IV collagen and early lethality in lysyl hydroxylase 3 null mice. Proc. Natl. Acad. Sci. USA 101, 14120-14125 (2004).

11. Salo, A. M. et al. A connective tissue disorder caused by mutations of the lysyl hydroxylase 3 gene. Am. J. Human. Genet. 83, 495-503 (2008).

12. Xiong, G., Deng, L., Zhu, J., Rychahou, P. G. \& Xu, R. Prolyl-4-hydroxylase a subunit 2 promotes breast cancer progression and metastasis by regulating collagen deposition. BMC Cancer 14, 1 (2014).

13. Pollard, J. W. Tumour-educated macrophages promote tumour progression and metastasis. Nat. Rev. Cancer 4, 71 (2004).

14. Cheon, D.-J. et al. A collagen-remodeling gene signature regulated by TGF- $\beta$ signaling is associated with metastasis and poor survival in serous ovarian cancer. Clin. Cancer Res. (2013).

15. Oudin, M. J. et al. Tumor Cell-driven extracellular matrix remodeling drives haptotaxis during metastatic progression. Cancer Discov. 6, 516-531 (2016).

16. Cheng, L. et al. Identification of genes with a correlation between copy number and expression in gastric cancer. BMC Med. Genom. 5, 14 (2012).

17. Nicastri, A. et al. N-glycoprotein analysis discovers new up-regulated glycoproteins in colorectal cancer tissue. J. Proteome Res. 13, 4932-4941 (2014).

18. Schiarea, S. et al. Secretome analysis of multiple pancreatic cancer cell lines reveals perturbations of key functional networks. J. Proteome Res. 9, 4376-4392 (2010).

19. Salo, A. M. et al. Lysyl hydroxylase 3 ( $\mathrm{LH} 3$ ) modifies proteins in the extracellular space, a novel mechanism for matrix remodeling. J. Cell. Physiol. 207, 644-653 (2006).

20. Patil, C. \& Walter, P. Intracellular signaling from the endoplasmic reticulum to the nucleus: the unfolded protein response in yeast and mammals. Curr. Opin. Cell Biol. 13, 349-355 (2001).

21. Kaufman, R. J. Stress signaling from the lumen of the endoplasmic reticulum: coordination of gene transcriptional and translational controls. Genes Dev. 13 1211-1233 (1999).

22. Schroder, M. \& Kaufman, R. J. The mammalian unfolded protein response. Annu. Rev. Biochem. 74, 739-789 (2005).

23. Rahmani, M. et al. The kinase inhibitor sorafenib induces cell death through a process involving induction of endoplasmic reticulum stress. Mol. Cell. Biol. 27, 5499-5513 (2007).

24. Kim, R., Emi, M., Tanabe, K. \& Murakami, S. Role of the unfolded protein response in cell death. Apoptosis 11, 5-13 (2006).

25. Basu, A. Involvement of protein kinase C-delta in DNA damage-induced apoptosis. J. Cell. Mol. Med. 7, 341-350 (2003)

26. Brodie, C. \& Blumberg, P. M. Regulation of cell apoptosis by protein kinase C delta. Apoptosis 8, 19-27 (2003).

27. Kumar, S. Caspase 2 in apoptosis, the DNA damage response and tumour suppression: enigma no more? Nat. Rev. Cancer 9, 897-903 (2009).

28. Hitomi, J. et al. Involvement of caspase-4 in endoplasmic reticulum stressinduced apoptosis and Abeta-induced cell death. J. Cell Biol. 165, 347-356 (2004).

29. Larroque-Cardoso, P. et al. Role of protein kinase C delta in ER stress and apoptosis induced by oxidized LDL in human vascular smooth muscle cells. Cell Death Dis. 4, e520 (2013).

30. Kuo, T. C., Huang, W. J. \& Guh, J. H. WJ9708012 exerts anticancer activity through PKC-a related crosstalk of mitochondrial and endoplasmic reticulum stresses in human hormone-refractory prostate cancer cells. Acta Pharmacol. Sin. 32, 89-98 (2011).

31. Zhao, M., Xia, L. \& Chen, G. Q. Protein kinase $c \delta$ in apoptosis: a brief overview. Arch. Immunol. Ther. Exp. 5, 361-372 (2012).

32. Song, Y. et al. Hypoxia-induced PLOD2 promotes proliferation, migration and invasion via PI3K/Akt signaling in glioma. Oncotarget 8, 41947-41962 (2017).

33. Gilkes, D. M. et al. Procollagen lysyl hydroxylase 2 is essential for hypoxiainduced breast cancer metastasis. Mol. Cancer Res. 11, 456-466 (2013).

34. Miyamoto, K. et al. Tumour-suppressive miRNA-26a-5p and miR-26b-5p inhibit cell aggressiveness by regulating PLOD2 in bladder cancer. Br. J. Cancer 115, 354-363 (2016).

35. Kurozumi, A. et al. Regulation of the collagen cross-linking enzymes LOXL2 and PLOD2 by tumor-suppressive microRNA-26a/b in renal cell carcinoma. Int. J. Oncol. 48, 1837-1846 (2016).

36. Hollern, D. P., Honeysett, J., Cardiff, R. D. \& Andrechek, E. R. The E2F transcription factors regulate tumor development and metastasis in a mouse model of metastatic breast cancer. Mol. Cell. Biol. 34, 3229-3243 (2014).

37. $\mathrm{Du}, \mathrm{H}$. et al. PLOD2 regulated by transcription factor FOXA1 promotes metastasis in NSCLC. Cell Death Dis. 8, e3143 (2017). 
38. Amodio, G. et al. Identification of a microRNA (miR-663a) induced by ER stress and its target gene PLOD3 by a combined microRNome and proteome approach. Cell Biol. Toxicol. 32, 285-303 (2016).

39. Sipila, L. et al. Secretion and assembly of type IV and VI collagens depend on glycosylation of hydroxylysines. J. Biol. Chem. 282, 33381-3338 (2007).

40. Shen, Q. et al. Barrier to autointegration factor 1, procollagen-lysine, 2oxoglutarate 5-dioxygenase 3, and splicing factor 3b subunit 4 as early-stage cancer decision markers and drivers of hepatocellular carcinoma. Hepatology 67, 1360-1377 (2018)
41. Tufo, G. et al. The protein disulfide isomerases PDIA4 and PDIA6 mediate resistance to cisplatin-induced cell death in lung adenocarcinoma. Cell Death Differ. 21, 685-695 (2014).

42. Ishida, S., McCormick, F., Smith-McCune, K. \& Hanahan, D. Enhancing tumorspecific uptake of the anticancer drug cisplatin with a copper chelator. Cancer Cell 17, 574-583 (2010)

43. Baek, J. H. et al. Kinesin light chain-4 depletion induces apoptosis of radioresistant cancer cells by mitochondrial dysfunction via calcium ion influx. Cell Death Dis. 9, 496 (2018). 